\title{
Tradução, adaptação e evidências de validade da Escala de Foco Regulatório (EFR]
}

\author{
Mary Sandra Carlotto \\ Sheila Gonçalves Câmara \\ Letícia Ribeiro S. Pinheiro
}

\section{RESUMO}

O Foco Regulatório diz respeito ao processo pelo qual as pessoas procuram alinhar-se, em termos de comportamentos e concepções, a metas ou padrões adequados de acordo com duas orientações motivacionais para atingir seus objetivos: foco para a promoção e foco para a prevenção. $O$ objetivo deste artigo foi analisar as evidências de validade da Escala de Foco Regulatório (EFR) no contexto brasileiro. Participaram do estudo 310 gestores recrutados por meio de instrumento de coleta de dados online. As evidências de validade foram verificadas por meio de análise fatorial confirmatória, alfa de Cronbach, ômega de McDonald e correlação de Pearson. Os resultados indicaram ajuste dos dados ao modelo teórico, adequada confiabilidade interna e validade convergente com a adição ao trabalho e ao engajamento ao trabalho. Assim, constatou-se que o instrumento é indicado para a avaliação do Foco Regulatório, no entanto, sugere-se a realização de novos estudos com amostras com outras categorias profissionais para verificação da estabilidade do instrumento.

Palavras-chave: Foco regulatório; Avaliação psicológica; Análise fatorial.

\section{ABSTRACT}

\section{Translation, adaptation and validity evidence of Regulatory Focus Scale [EFR]}

The Regulatory Focus refers to the process by which people seek to align themselves, in terms of behaviors and concepts, to appropriated goals or standards according to two motivational orientations to achieve their goals: promotion focus and prevention focus. The purpose of this article was to analyze the validity pieces of evidence of the Regulatory Focus Scale (EFR) in the Brazilian context. The participants were 310 managers recruited through an online data collection tool. Pieces of evidence of validity were verified through confirmatory factor analysis, Cronbach alpha, McDonald's omega, and Pearson's correlation. Results indicate adjustment of the data to the theoretical model, adequate internal reliability, and convergent validity with the workaholism and engagement to work. Thus, the instrument is indicated for the assessment of Regulatory Focus however, it is suggested to accomplish new studies with samples with other professional categories to test its stability.

Keywords: Regulatory focus; Psychological assessment; Factor analysis.

O conceito de Foco Regulatório (FR), proposto por Higgins (1997), tem se mostrado importante para explicar o processo motivacional em contextos laborais (Brenninkmeijer et al., 2010) e tem recebido crescente atenção em psicologia organizacional (Lanaj et al., 2012) devido ao seu potencial para análise de diversos resultados organizacionais (Kark et al., 2018).

A autorregulação é fundamental para o conceito do FR uma vez que se refere ao funcionamento adaptativo. As pessoas precisam regular suas cognições e comportamentos durante a busca de objetivos (Higgins, 1997). Trata-se de um processo pelo qual as pessoas procuram alinhar-se, em termos de comportamentos e concepções, a metas ou padrões adequados (Crowe \& Higgins, 1997).

\section{Sobre os autores}

M. S. C.

http://orcid.org/0000-0003-

2336-5224

Universidade do Vale do Rio dos

Sinos (UNISINOS)

mscarlotto@unisinos.br

S. G. C.

http://orcid.org/0001-6761-

7644

Universidade Federal de Ciências da Saúde de Porto Alegre (UFCSPA)

sheila.gcamara@gmail.com

L. R. S. P.

https://orcid.org/0000-00023401-860X

Universidade Regional Integrada do Alto Uruguai e das Missões (URI) - Erechim, RS

leticia.rsp@terra.com.br

\section{Direitos Autorais}

Este é um artigo de acesso aberto e pode ser reproduzido livremente, distribuído, transmitido ou modificado, por qualquer pessoa desde que usado sem fins comerciais. $O$ trabalho é disponibilizado sob a licença Creative Commons CC-BY-NC.

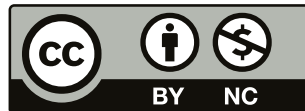




\section{H. INTERACÃO EM LF PSICOLOGIA}

Os indivíduos diferem em como se aproximam do prazer e tentam evitar a dor, sendo que estas diferenças se manifestam em duas orientações motivacionais distintas que regem a forma como as pessoas perseguem objetivos: foco voltado para a promoção e foco voltado para a prevenção (Higgins, 1997). Pessoas focadas na promoção são motivadas por necessidades de crescimento, desenvolvimento e realização, de forma a aproximar-se de situações ideais baseadas em desejos e aspirações de como gostariam de ser. Quando o foco é a prevenção, as pessoas respondem a necessidades de segurança e buscam adequar-se às normas baseadas em deveres e responsabilidades. A busca por ganhos e sucesso predomina para pessoas com foco na promoção, e a vigilância e comportamentos para evitar perdas e fracasso caracterizam as pessoas com foco em prevenção (Brockner \& Higgins, 2001).

Revisão sistemática da literatura realizada por Lanaj et al. (2012) identificou várias relações significativas entre foco de promoção e prevenção e comportamentos de trabalho. 0 foco na promoção foi positivamente relacionado a desempenho eficaz e inovador das tarefas, maior engajamento e satisfação no trabalho e comprometimento afetivo organizacional. O foco na prevenção evidenciou associação positiva com um desempenho de tarefas realizadas de acordo com procedimentos já instituídos e seguros e foi associado negativamente com a satisfação no trabalho e positivamente ao comprometimento organizacional normativo.

0 conceito tem sido utilizado em investigações que buscam sua associação com a adição ao trabalho (Van Beek et al., 2014), engajamento no trabalho (Vasanthi \& Geetha, 2019), liderança (González-Cruz et al., 2019), emoções no trabalho (Geng et al., 2018), tomada de decisão (Peng et al., 2019; Vohs et al., 2014), desempenho individual e de grupos (Johnson \& Wallace, 2011; Wallace et al., 2008), comportamento proativo e desenvolvimento de carreira (Strauss et al., 2012; Van Vianen et al., 2012) e inovação e criatividade (Li et al., 2018). Também tem sido utilizado em estudos sobre sua relação com comportamentos de saúde (Li \& Hu, 2019; Liang et al., 2012), ansiedade (Llewellyn et al., 2013) e adesão a tratamento (Fuglestad et al., 2008).

Quanto aos instrumentos de avaliação do Foco Regulatório, duas recentes revisões de literatura (Gorman et al., 2012: Johnson et al., 2015) identificaram que a medida mais utilizada foi a General Regulatory Focus Measure (GRFM) de Lockwood et al. (2002). Tal medida inicialmente foi elaborada para avaliação do foco motivacional direcionado a objetivos acadêmicos, tendo sido adaptada e utilizada para avaliar o construto em estudos organizacionais (Brenninkmeijer et al., 2010; Walker, 2012). Para tanto, foi realizada a alteração dos termos "objetivos acadêmicos" para "objetivos profissionais". A escala avalia como o sujeito regula seu foco para obtenção de objetivos em duas dimensões: foco na promoção (em que medida uma pessoa está focada na obtenção de resultados positivos) e foco na prevenção (em que medida uma pessoa está focada em evitar resultados negativos).

Avaliar o FR em trabalhadores tem sido referida como uma importante estratégia de gestão devido ao seu caráter disposicional e maleável. Neste sentido, pode subsidiar atividades de seleção, treinamento e desenvolvimento, avaliação de desempenho, promoção e remuneração (Johnson et al., 2015). Em atividades que envolvem atenção a riscos e vigilância como controladores de tráfego aéreo, agentes de segurança e corretores de investimento financeiro, o foco na prevenção pode ser importante, e nas que envolvem inovação, planejamento e criação é desejável o foco na promoção (Johnson et al., 2015; Lanaj et al., 2012). Programas de intervenção podem auxiliar gestores e trabalhadores a ajustar o foco de acordo com a atividade e contexto de trabalho, principalmente quando determinadas atividades apresentam conflito entre velocidade de produção e segurança (Wallace \& Chen, 2006). Avaliar o FR especificamente em gestores é relevante na medida em que sua motivação está relacionada ao estilo de liderança, sua capacidade de influenciar positivamente seus liderados e obter o melhor desempenho seu e de sua equipe (Kark et al., 2007).

No Brasil existem dois estudos que abordem o foco regulatório em nível teórico (Mantovani et al., 2012; Mantovani et al., 2015); não se identificou, nas principais bases de dados (Biblioteca Virtual em Saúde, Pepsic, Pubmed e Scielo), estudos que utilizassem a escala e que avaliassem suas propriedades psicométricas. Pelo exposto, o objetivo deste estudo foi analisar as evidências de validade da EFR em uma amostra de gestores.

\section{MÉTODO}

\section{PARTICIPANTES}

A amostra foi constituída por 310 trabalhadores que ocupam cargos de gestão como proprietários $(13,2 \%)$, diretores $(9,4 \%)$ e gerentes $(22,9 \%)$, líderes de setores (38\%) e consultores $(16,5 \%)$, dos setores econômicos da indústria $(25,2 \%)$, comércio $(29,1 \%)$ e serviços (45,6\%). A maioria é composta de homens $(54,1 \%)$, com união estável $(71,3 \%)$, que possuem fiIhos (53,5\%). A idade dos participantes variou de 20 a 64 anos $(\mathrm{M}=38,5 ; \mathrm{DP}=9,4)$. Em média, atuam profissionalmente há 18,4 anos (DP $=9,8$ ) com uma variação de 01 a 48 anos e no cargo de gestão nove anos (DP = 8). A maioria trabalha em empresa privada $(88,5 \%)$ e possui uma remuneração entre $R \$$ 1.734 e R\$ 7.475 (50,5\%) de acordo com a classificação da Fundação Getúlio Vargas.

\section{INSTRUMENTOS}

Questionário sociodemográfico (sexo, idade, situação conjugal, presença de filhos, formação) e laboral (carga ho- 


\section{H* INTERACÃO EM PSICOLOGIA}

rária semanal, tempo de atuação profissional, tempo no cargo de gestor, tipo de empresa, remuneração/mensal).

Escala Geral de Focus Regulatório, desenvolvida por Lockwood et al. (2002) e adaptada para contextos organizacionais (vide próximo tópico). Avalia como o sujeito regula seu foco para obtenção de metas: foco na promoção, em que medida uma pessoa está focada na obtenção de resultados positivos ( 9 itens; $a=0,81$ ) e foco na prevenção, em que medida uma pessoa está focada em evitar resultados negativos (9 itens; $a=0,75)$. A escala é avaliada pelo sistema de pontuação tipo Likert, variando de 1 (muito falso a meu respeito) a 5 (muito verdadeiro a meu respeito).

Dutch Work Addiction Scale (DUWAS) de Schaufeli et al. (2006), adaptada para o uso no Brasil por Carlotto e Del Líbano (2010) para avaliação da Adição ao Trabalho. A escala possui 10 itens que avaliam o construto em duas dimensões: o Trabalho Compulsivo (cinco itens; $a=0,70$ ) e o Trabalho Excessivo (cinco itens; $a=0,74$ ). No total, constitui-se de dez itens avaliados por uma escala tipo Likert, variando de 1 (nunca) a 4 (todos os dias).

Utrecht Work Engagement Scale (UWES) de Schaufeli e Bakker (2004), adaptada para o Brasil por Vazquez et al. (2015) para avaliação do engajamento no trabalho. Constituída por 17 itens, que compõem 3 dimensões: Vigor (seis itens; $a=80$ ); Dedicação (cinco itens; $a=89$ ), Absorção (seis itens; $a=72$ ), além de um escore geral do constructo $(a=0,81)$ (Schaufeli \& Bakker, 2004; Bakker et al., 2008; Salanova \& Schaufeli, 2009). Os itens são avaliados por uma escala tipo Likert de sete categorias que varia de 0 (nunca) a 6 (sempre).

\section{TRADUÇÃO E ADAPTAÇÃO}

0 procedimento de validação do instrumento foi autorizado por seus autores, representados pela primeira autora, Prof. Dr. Penelope Lockwood. A partir da versão original, em inglês, a versão brasileira da EFR seguiu os padrões metodológicos recomendados pela Comissão Internacional de Testes (ITC) para adaptação adequada de instrumentos a outras culturas (Muñiz \& Hambleton, 2000). A tradução e a retrotradução foram realizadas por três tradutores com domínio do idioma inglês e português do Brasil. Na avaliação da equivalência semântica, verificou-se que o significado referencial apresentou bons resultados, tendo em vista que os itens obtiveram, nas retrotraduções entre 90,0\% e 100\% de concordância nesse critério (Reichenheim \& Moraes, 2007).

A versão final em português do Brasil foi submetida a uma testagem piloto, para fins de avaliação semântica, com 10 trabalhadores, não pertencentes à população em estudo. Foi solicitado aos participantes que manifestassem aspectos relativos ao entendimento de palavras e enunciados. Nessa etapa houve necessidade de modificação de três palavras em dois itens.

\section{PROCEDIMENTOS DE COLETA E ANÁLISE DE DADOS}

Os dados do estudo foram coletados entre março e agosto de 2015, por meio de pesquisa on-line, mediante convite via e-mail. Os participantes que aceitaram o convite indicaram sua concordância ao Termo de Consentimento Livre e Esclarecido, de acordo com as diretrizes éticas da Resolução 466 do Conselho Nacional de Saúde no tocante à realização de pesquisa com seres humanos (CONEP, 2012). Após, os participantes eram direcionados à plataforma com os instrumentos e o tempo médio de respostas foi de 40 minutos. 0 estudo foi aprovado pelo Comitê de Ética em Pesquisa da Pontifícia Universidade Católica do Rio Grande Do Sul - PUC/RS (CAAE 46803415.0.0000.5336).

0 recrutamento dos participantes foi utilizado por meio da técnica do Respondent Driven Sampling (RDS), que combina a amostragem em bola de neve (snowball sampling; Goodman, 1961) e a utilização de redes de contatos como uma forma de compensar a amostragem não aleatória (Heckathorn, 1997). Assim, os primeiros participantes ( $1^{\text {a }}$ onda) enviaram o convite para novos participantes ( $2^{\mathrm{a}}$ onda), até que se alcançasse o tamanho desejado da amostra (Goel \& Salganik, 2009). A primeira onda envolveu a rede de contatos das pesquisadoras e de participantes do grupo de pesquisa, alunos de iniciação científica, mestrandas e doutorandas, redes sociais e por mapeamento de guias telefônicos virtuais. Nessa, foram contatadas em torno de 300 pessoas. A segunda e as ondas subsequentes ocorreram a partir das sementes iniciais, isto é, a rede de contados dos primeiros participantes. Essa estratégia torna a amostra independente dos contatos iniciais. Após as sucessivas ondas, a composição amostral torna-se relativamente estável (Heckathorn, 2002; Salganik \& Heckathorn, 2004).

$\mathrm{O}$ banco de dados foi digitado e, posteriormente, analisado no pacote estatístico SPSS, versão 17.0. Realizaram-se análises descritivas de caráter exploratório a fim de avaliar, no banco de dados, a distribuição dos itens e identificação de extremos. Foi conduzida uma análise fatorial pelo método de extração dos eixos principais, rotação varimax para verificar a distribuição fatorial do instrumento. 0 critério de retenção de fatores foi a análise paralela através da permutação aleatória dos valores amostrais (Timmerman \& Lorenzo-Seva, 2011).

Foi realizada Análise Fatorial Confirmatória (AFC), método de máxima verossimilhança, utilizando-se o programa estatístico AMOS 21.0. A confiabilidade foi avaliada pelo método do alfa de Cronbach (a) e o estimador ômega de McDonald ( $\omega t)$, que foi utilizado para suprir limitações do a (Dunn et al., 2013). Para evidência de validade de construto foi utilizada a prova de correlação de Pearson entre as dimensões da EFR, Foco na Prevenção e Foco na Promoção e as dimensões de Adição ao Trabalho (Trabalho Excessivo e Trabalho Compulsivo) e de Engajamento ao Trabalho (Vigor, Dedicação e Absorção). 


\section{RESULTADOS}

\section{ANÁLISE FATORIAL EXPLORATÓRIA}

A análise fatorial exploratória foi realizada para identificação da distribuição fatorial do instrumento. Uma primeira análise indicou quatro fatores, de acordo com seus autovalores. A análise paralela indicou que deveriam ser retidos dois fatores. Em nova análise fatorial exploratória, delimitada em dois fatores, a medida KMO (Kaiser-Meyer-Olkin) foi de 0,81
Foi testado, inicialmente, o modelo original, com 18 itens e dois fatores. Esse modelo, no entanto, não apresentou indicadores satisfatórios: $\chi 2=659,082, p=0,000, \mathrm{gl}=134, \chi 2 / \mathrm{gl}=$ 4,91 , Normed Fit Index (NFI) =0,683, Goodness Fit Index (GFI) $=0,816$, Comparative Fit Index $(\mathrm{CFI})=0,728$, e RMSEA = 0,109. 0 modelo somente indicou índices adequados após a eliminação de 6 itens que apresentaram baixa carga fatorial $(\lambda=0,30)$ e realização de correlação entre os erros dos itens 2 e 4, 7 e 8 , 16 e 17, 17 e 18 (todos de Foco na Prevenção; Tabela 1).

Tabela 1. Modelo ajustado para a Escala de Foco Regulatório (EFR)

\begin{tabular}{lcccccc}
\hline Modelo & $\chi 2$ & gl & $\chi 2 / g \mid$ & GFI & CFI & $\operatorname{RMSEA~}(90 \%$ Cl \\
\hline$M_{1}$ (18 itens) & 659,082 & 134 & 4,91 & 0,816 & 0,728 & $0,109[0,101-0,117]$ \\
$M_{2}$ (12 itens) & 139,491 & 49 & 2,84 & 0,939 & 0,920 & $0,075[0,060-0,090]$ \\
\hline
\end{tabular}

e o Teste de Esfericidade de Bartlett apresentou qui-quadrado aproximado de 1119,13 e nível de significância de 0,00, indicando que a matriz correlacional não era uma matriz identidade. A variância explicada obtida foi de $35,6 \%$ dos dados. 0 primeiro fator ficou composto de 11 itens e o segundo, de 7 itens. Quanto às cargas fatoriais, os itens 1 e 15 (fator 1) e 4 (fator 2) apresentaram carga fatorial inferior a 0,30.

\section{ANÁLISE FATORIAL CONFIRMATÓRIA}

Para avaliar o modelo teórico de dois fatores, de acordo com Lockwood et al. (2002), foi realizada análise fatorial confirmatória. Foi utilizado o programa AMOS 21.0, adotando-se o estimador máxima verossimilhança, que é adequado para amostras entre 150 e 400 participantes, as quais não interferem na sensibilidade do método (Hair et al., 2009). Foram considerados os seguintes índices estatísticos: $\chi 2$ (qui-quadrado), que comprova a probabilidade de um modelo se ajustar aos dados; Razão x2/gl (graus de liberdade), que representa uma bondade de ajuste subjetiva (Byrne, 2010); Normed fit in$\operatorname{dex}(\mathrm{NFI})$, que analisa a discrepância entre o valor de qui-quadrado do modelo hipotetizado e o valor de qui-quadrado do modelo nulo (Tabachnick \& Fidell, 2012); Goodness of fit index (GFI), que mede a variabilidade explicada pelo modelo ser afetado pelo tamanho da amostra (Saris \& Stronkhorst, 1984); Comparative fit index (CFI), que representa o ajuste proporcional adicionado ao modelo pela comparação com um modelo de referência, baseado em um modelo nulo no qual todas as variáveis observáveis não são correlacionadas (Hu \& Bentler, 1999); e Root mean square error of approximation (RMSEA), que corresponde à quantidade de erro de aproximação populacional numa matriz de covariância (Browne \& Cudeck, 1993).
O modelo final (Figura 1) alcançou boa adequação aos dados para a amostra em todos os índices de ajuste considerados: $\chi 2=139,491, p=0,000, \mathrm{gl}=49, \chi 2 / g l=2,84$, Normed Fit Index $(\mathrm{NFI})=0,884$, Goodness Fit Index $(\mathrm{GFI})=0,939$, Comparative Fit Index $(\mathrm{CFI})=0,920$ e RMSEA $=0,075$. Devido à prova $\chi 2$ ser sensível ao tamanho da amostra, calculou-se a razão entre seu valor e os graus de liberdade. Entretanto, como não existe um valor crítico exato para decidir sobre a adequação do modelo, são aceitos índices iguais ou inferiores a 5. Neste estudo, o valor obtido indica que o modelo se ajusta aos dados $(\chi 2 / \mathrm{gl}=$ 2,84 ). A quantidade relativa de variância explicada pelo modelo $(\mathrm{GFI}=0,939)$ foi suficiente e o ajuste do modelo resultou adequado ao considerar o erro de aproximação aos valores da matriz de covariância da população (RMSEA $=0,075)$, assim como os índices de ajuste relativo do modelo ( $\mathrm{CFI}=0,92$; Byrne, 2010).

\section{ESTATÍSTICA DESCRITIVA}

As estatísticas descritivas da EFR podem ser observadas na Tabela 2. 0 fator que obteve a média mais elevada foi o de Foco na Promoção $(M=3,95)$ e os itens com maiores médias foram o 16 (Em geral, eu estou focado em conseguir resultados positivos na minha vida; $\mathrm{M}=4,37$ ), 17 (Frequentemente eu me imagino vivenciando situações boas e que eu espero venham a acontecer comigo; $M=4,18$ ) e 6 (Eu geralmente me foco no sucesso que eu espero atingir no futuro; $M=4,06$ ), que pertencem à mesma dimensão. Em relação à correlação item-total corrigida, a maioria dos itens obteve valores superiores a $r=0,40$.

Os valores de assimetria obtidos nos itens do EFR permitem afırmar que, em geral, apresentam uma distribuição normal. 0 valor mais elevado de assimetria foi obtido no item $17($ As $=-0,89)$, pertencente à escala Foco na Promoção. Nas 


\section{H NTERAC̄öEM ET PSICOLOGIA}

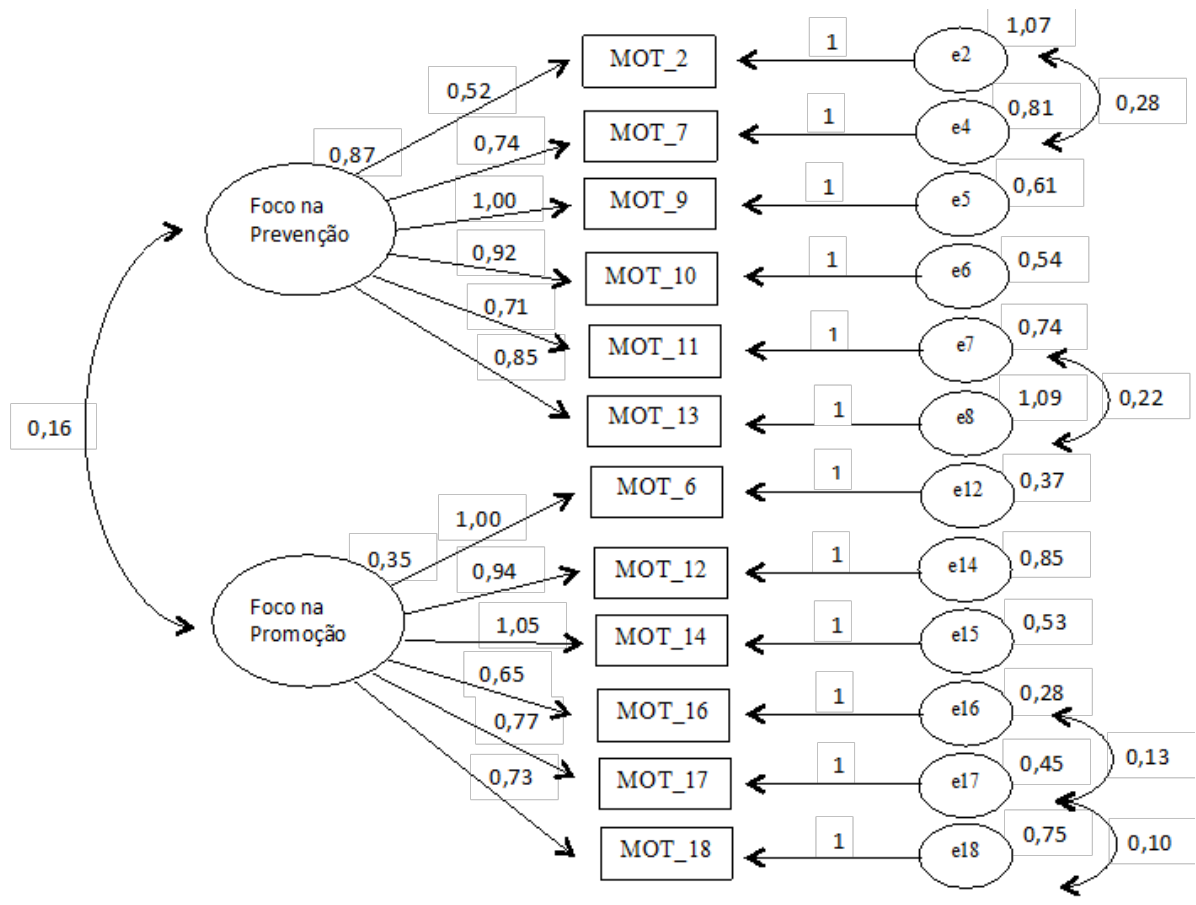

Figura 1. Resultados do modelo fatorial hipotetizado para a Escala Focus Regulatório (EFR) $(n=310)$.

duas dimensões da EFR, o valor do índice de assimetria encontra-se dentro do intervalo \pm 1 , o que possibilita afirmar que apresentaram uma distribuição normal. Os valores do alfa de Cronbach e do ômega de McDonald foram, respectivamente, 0,81 e 0,88 para o fator Foco na prevenção, e de 0,76 e 0,81 para o fator Foco na Promoção (Tabela 2).

\section{VALIDADE CONVERGENTE}

Foi realizada análise de correlação entre as dimensões da EFR, Foco na Prevenção e Foco na Promoção com as dimensões de Adição ao Trabalho (Trabalho Excessivo e Trabalho Compulsivo) e dimensões do Engajamento ao Trabalho (Vigor, Dedicação e Absorção). Os resultados revelaram, conforme o esperado, relação positiva entre a dimensão Foco na Promoção com as dimensões do engajamento e entre o Foco na Prevenção com a Adição ao Trabalho (Tabela 3).

\section{DISCUSSÃO}

O objetivo deste artigo foi analisar as evidências de validade da Escala de Foco Regulatório (EFR) no contexto brasileiro em uma amostra de trabalhadores com a função de gestão. Os resultados corroboraram o modelo teórico de dois fatores, de acordo com o original da escala (Lockwood et al., 2002).

A partir dos resultados de adequação das propriedades psicométricas, pode-se afirmar que o modelo final obtido está adequado para avaliação do FR. A técnica de análise paralela para retenção fatorial permitiu a identificação de um modelo bifatorial que apresentou uma distribuição semântica dos itens similar a do modelo original da escala (Lockwood et al., 2002). A análise fatorial exploratória, no entanto, foi utilizada apenas como subsídio para identificação do número de fatores e não para a avaliação de um modelo específico para a amostra em estudo.

Neste estudo foi avaliada a versão original da escala (Lockwood et al., 2002) e a Análise Fatorial Confirmatória corroborou a estrutura teórica inicial de dois fatores. A correlação entre as dimensões foi baixa, porém significativa $(r=0,16 ; p \leq$ $0,01)$. No presente estudo, foram eliminados 6 itens que apresentaram baixa carga fatorial $(\lambda=0,30)$. Assim, a escala final ficou constituída por 12 itens, 6 em cada dimensão. 0 modelo final obtido apresentou boa adequação aos dados para a amostra em todos os índices de ajuste considerados (Tabachnick \& Fidell, 2012). Com estes valores, pode-se concluir que o modelo de equações estruturais apresentou um ajuste global aos dados observados e corroborou o modelo teórico do instrumento com a exclusão de seis itens na amostra em estudo.

Os valores da correlação item-total corrigida foram elevados, evidenciando que cada dimensão da escala pode ser considerada com uma função linear dos itens que a compõem. As duas dimensões demonstram ser independentes assim como proposto pelo modelo teórico de Higgins (1997) e do resultado identificado em meta-análise realizada por Gorman et al. (2012). 
Tabela 2. Estatistica descritiva e consistência interna dos itens e dimensões da Escala Foco Regulatório $(E F R)(n=310)$

\begin{tabular}{lccccc}
\hline Subescala/Item & $\mathrm{M}(\mathrm{DP})$ & $\begin{array}{c}\text { Correlação item-total } \\
\text { corrigida }\end{array}$ & Assimetria & $\begin{array}{c}\text { Alfa } \\
\text { sem item }\end{array}$ & $\begin{array}{c}\text { Omega } \\
\text { sem item }\end{array}$ \\
\hline Foco na prevenção & $3,16(0,84)$ & & $-0,07$ & & 0,81 \\
2 & $3,77(1,14)$ & 0,44 & $-0,79$ & 0,81 & 0,79 \\
7 & $3,62(1,13)$ & 0,60 & $-0,53$ & 0,78 & 0,77 \\
9 & $2,93(1,22)$ & 0,66 & 0,12 & 0,76 & 0,78 \\
10 & $3,34(1,13)$ & 0,61 & $-0,26$ & 0,78 & 0,79 \\
11 & $2,72(1,08)$ & 0,59 & 0,27 & 0,78 & 0,79 \\
13 & $2,62(1,31)$ & 0,57 & 0,39 & 0,79 & 0,82 \\
Total & $3,18(0,84)$ & & & 0,81 & \\
& & & & \\
Foco na Promoção & $3,95(0,60)$ & & $-0,39$ & & 0,72 \\
6 & $4,03(0,85)$ & 0,54 & $-0,60$ & 0,71 & 0,76 \\
12 & $3,44(1,07)$ & 0,42 & $-0,28$ & 0,75 & 0,74 \\
14 & $3,80(0,95)$ & 0,53 & $-0,48$ & 0,71 & 0,73 \\
16 & $4,37(0,65)$ & 0,55 & $-0,75$ & 0,72 & 0,72 \\
17 & $4,18(0,81)$ & 0,56 & $-0,89$ & 0,71 & 0,76 \\
18 & $3,88(0,96)$ & 0,44 & $-0,54$ & 0,74 & 0,81 \\
Total & $3,96(0,59)$ & & & 0,76 & \\
\hline
\end{tabular}

Tabela 3. Matriz de correlação entre as dimensões de estudo $(n=310)$

\begin{tabular}{lccccccc}
\hline Variáveis & 1 & 2 & 3 & 4 & 5 & 6 & 7 \\
\hline 1. Foco na Promoção & 1 & & & & & & \\
2. Foco na Prevenção & $0,15 * *$ & 1 & & & & & \\
3. Trabalho Excessivo & 0,06 & $0,17 * *$ & 1 & & & & \\
4. Trabalho Compulsivo & 0,10 & $0,24 * *$ & $0,58^{* *}$ & 1 & & & \\
5. Vigor & $0,25 * *$ & $-0,08$ & 0,06 & $0,14 * *$ & 1 & & \\
6. Dedicação & $0,31 * *$ & $-0,08$ & 0,07 & 0,09 & $0,80 * *$ & 1 & \\
7. Absorção & $0,24 * *$ & 0,05 & $0,30 * *$ & $0,32 * *$ & $0,74 * *$ & $0,70 * *$ & 1 \\
\hline$* * p<0,001$
\end{tabular}

Os valores obtidos para assimetria indicam adequação dos dados, uma vez que foi identificada variação de $-0,07$ a 0,39 . Valores de assimetria aceitáveis estão contidos no intervalo de \pm 1 para que se possa concluir que o modelo tem uma distribuição normal (Miles \& Shevlin, 2005). Os valores obtidos de alfa de Cronbach e ômega, satisfatórios em cada dimensão (Tabachnick \& Fidell, 2012), aliados à coerência semântica do conjunto de itens, permitem considerar que todos contribuem para o estabelecimento do construto no que tange à avaliação do FR.

A validade convergente confirmou o estudo realizado por Van Beek et al. (2014), demonstrando associação positiva entre a dimensão Foco na Promoção e as dimensões do engajamento ao trabalho. Da mesma forma, foi identificada relação positiva entre o Foco na Prevenção e as dimensões de Adição ao Trabalho. Embora as correlações obtidas sejam baixas, todas apresentaram $p<0,001$. Os construtos utilizados para a análise não são correlatos das dimensões da EFR, no entanto, puderam servir como padrão de análise de acordo com os pressupostos teóricos referentes às definições de foco na promoção e foco na prevenção (Brockner \& Higgins, 2001).

Nesse sentido, ressalta-se que os diversos aspectos de validade do instrumento apresentaram resultados satisfatórios, indicando que a EFR possui propriedades psicométricas adequadas para o estudo do FR em gestores. $O$ instrumento pode, assim, contribuir para a avaliação do construto uma vez que este é um importante aspecto de gestão organizacional e desenvolvimento de carreira (Zivnuska et al., 2017).

Como limitação do estudo destaca-se a escolaridade em nível de graduação e pós-graduação dos participantes. Tal 


\section{M." INTERACÃO EM PSICOLOGIA}

característica pode ser devido ao fato de a coleta ter sido online. A utilização de tecnologias de informação e comunicação demanda acessibilidade e familiaridade com esse tipo de recurso, o que é compatível com maiores níveis educacionais (Heen et al., 2014). A partir dos resultados encontrados e das limitações do presente estudo, sugere-se que sejam conduzidos novos estudos de validade da EFR com amostra aleatória estratificada, a fim de verificar o comportamento do instrumento de acordo com o sexo e escolaridade. Além da avaliação dessas variáveis, a investigação com trabalhadores de diferentes categorias profissionais em diferentes contextos econômicos e socioculturais, contribuirá para a compreensão da estabilidade do instrumento.

\section{CONTRIBUIÇÃO DE CADA AUTOR}

Mary Sandra Carlotto foi responsável pela administração do projeto, conceitualização, redação - preparação do rascunho original e redação - revisão e edição.

Sheila Gonçalves Câmara foi responsável pela análise formal dos dados e redação - preparação do rascunho original.

Letícia Ribeiro foi responsável pela conceitualização, tabulação dos dados e redação - preparação do rascunho original.

\section{DECLARAÇÃO DE CONFLITOS DE INTERESSES}

Os autores declaram que não há conflitos de interesse neste artigo.

\section{REFERENNCIAS}

Brenninkmeijer, V., Demerouti, E., Le Blance, P.M., \& Van Emmerik, I. J. H. (2010). Regulatory focus at work: the moderating role of regulatory focus in the job demands-resources model. Career Development International, 15, 708-728. https://doi.org/10.1108/13620431011094096

Brockner, J., \& Higgins, E. T. (2001). Regulatory focus theory: Implications for the study of emotions at work. Organizational Behavior and Human Decision Processes, 86, 35-66. https://doi.org/10.1006/obhd.2001.2972

Byrne, B. M. (2010). Structural equation modeling with AMOS. Basic concepts, appli- cations and programming (2nd ed.). Routledge.

Conselho Nacional de Saúde (Brasil) - CONEP. (2012). Resolução $n{ }^{\circ}$ 466/212. http://www.conselho.saude.gov.br/ web_comissoes/conep/index.html.

Crowe, E., \& Higgins, E.T. (1997). Regulatory focus and strategic inclinations: Promotion and prevention in decision-making. Organizational Behavior and Human Decision Processes, 69, 117-132.
Dunn, T. J., Baguley, T., \& Brunsden, V. (2013). From alpha to omega: A practical solution to the pervasive problem of internal consistency estimation. British Journal of Psychology, 105(3),399-412. https://doi.org/10.1111/bjop.12046

Fuglestad, P. T., Rothman, A. J., Jeffery, R.W., \& Kaplan, R. M. (2008). Getting there and hanging on: the effect of regulatory focus on performance in smoking and weight loss interventions. Health Psychology, 27(3), 260-270.

Geng, Z., Li, C., Bi, K., Zheng, H., \& Yang, X. (2018). Motivating service employee creativity: regulatory focus and emotional labour. Journal of Service Theory and Practice, 28(2), 228-249. https://doi.org/10.1108/JSTP-11-2016-0214

Goel, S., \& Salganik, M. J. (2009). Respondent-driven sampling as Markov chain Monte Carlo. Statistics in Medicine, 28, 2202-2229. https://doi.org/10.1002/sim.3613

González-Cruz, T. F., Botella-Carrubib, D., \& Martínez-Fuentesa, C. M. (2019). Supervisor leadership style, employee regulatory focus, and leadership performance: A perspectivism approach. Journal of Business Research,101, 660667. https://doi.org/10.1016/j.jbusres.2019.01.065

Gorman, C. A., Meriac, J. P., Overstreet, B. L., Apodaca, S., Mclntyre, A. L., Park, P., \& Godbey, J. N. (2012). A meta-analysis of the regulatory focus nomological network: work-related antecedents and consequences. Journal of Vocational Behavior, 80, 160-172. https://doi.org/10.1016/j.jvb.2011.07.005

Hair, J. F., Anderson, R. E., Tatham, R. L., \& Black, W. C. (2007). Análisis Multivariante. Pearson - Prentice Hall.

Heckathorn, D. D. (1997). Respondent-driven sampling: a new approach to the study of hidden populations. Social Problems, 44, 174-199. https://doi.org/org/10.2307/309694

Heckathorn, D. D. (2002). Respondent-Driven Sampling II: Deriving Valid Population Estimates from Chain-Referral Samples of Hidden Populations. Social Problems, 49, 11-34. https://doi.org/10.1525/sp.2002.49.1.11

Heen, M. S. J., Lieberman, J. D., \& Miethe, T. D. (2014). A comparison of different online sampling approaches for generating national samples. Research in Brief. Center for Crime and Justice Policy. University of Nevada. http://dx.doi. org/10.13140/RG.2.2.24283.62243

Higgins, E. T. (1997). Beyond pleasure and pain. American Psychologist, 52, 1280-1300.

Johnson, P. D., Smith, M. B., Wallace, J. C. Aaron D. H., \& Baron R.A. (2015). A review of multilevel regulatory focus in organizations. Journal of Management, 41(5), 1501-1529. https://doi.org/10.1177/0149206315575552

Johnson, P. D., \& Wallace, J. C. (2011). Increasing individual and team performance in an organizational setting through the situational adaptation of regulatory focus. Consulting Psychology Journal: Practice and Research, 63, 190-201. https://doi.org/10.1037/a0025622 


\section{H MTERAC̄OOEM ET. PSICOLOGIA}

Kanfer, R., Frese, M., \& Russell E. J. (2017). Motivation related to work: A century of progress. Journal of Applied Psychology, 102(3), 338-355. https://doi.org/10.1037/apl0000133

Kark, R., \& Van Dijk, D. (2007). Motivation to lead, motivation to follow: the role of the self- regulatory focus in leadership processes. Academy of Management Review 32(2), 500-528. https://doi.org/10.2307/20159313

Kark, R., Van Dijk, D., \& Vashdi, D. R. (2018). Motivated or demotivated to be creative: the role of self-regulatory focus in transformational and transactional leadership processes. Applied Psychology: An International Review, 67(1), 186-224. https://doi.org/10.1111/apps.12122

Lanaj, K., Chang, C-H., \& Johnson, R E. (2012). Regulatory focus and work-related outcomes: a review and meta-analysis. Psychological Bulletin, 138(5), 998-1034. http:// dx.doi.org/10.1037/a0027723

Li, C-R Li, C-X., \& Lin, C-J. (2018). How and when team regulatory focus influences team innovation and member creativity, Personnel Review, 47(1), 95-117, https://doi. org/10.1108/PR-09-2016-0236

$\mathrm{Li}, \mathrm{A}-\mathrm{S}-\mathrm{K}, \& \mathrm{Hu}, \mathrm{T}-\mathrm{Y}$ (2019). Are immediate-oriented people unlikely to adopt a healthy lifestyle? The moderation effect of prevention focus. Personality and Individual Differences, 142(1), 276-281. https://doi.org/10.1016/j.paid.2018.10.004

Liang, H. L., Kao, Y.T., \& Lin, C. C. (2013). Moderating effect of regulatory focus on burnout and exercise behavior. Perceptual and Motor Skills, 117(3), 696-708.

Llewellyn, N., Dolcos, S., Jordan., A. D., Rudolph, K., \& Dolcos, F. (2013). Reappraisal and suppression mediate the contribution of regulatory focus to anxiety in healthy adults. Emotion,13(4), 610-615. https://doi.org/10.1037/a0032568

Lockwood, P., Jordan, C. H., \& Kunda, Z. (2002). Motivation by positive or negative role models: regulatory focus determines who will best inspire us. Journal of Personality and Social Psychology, 83(4), 854-864. https://doi. org/10.1037/0022-3514.83.4.854

Mantovani, D., Barboza, M. M., Viacava, J. J. C., \& Prado, P. H. M. (2015). Quando forças opostas aumentam a intenção de compra? foco motivacional e mensagens de comunicação. Revista de Administração Contemporânea, 19, (n.spe2), 178-196. https://doi.org/10.1590/1982-7849rac20151579

Mantovani, D., Korelo, J. C., \& Prado, P. H. M. (2012). Como você fez sua escolha?: o papel do foco motivacional sobre a tendência a inovar. Revista de Administração Contemporânea, 16(2), 179-199. https://doi.org/10.1590/S141565552012000200002

Miles, J., \& Shevlin, M. (2005). Applying regression and correlation. A guide for students and researchers. Sage.

Peng, J., Cao, F., Zhang, Y., Cao, Y., Zhang, Y., Zhu, X. \& Miao, D. (2019). Reflections on motivation: How regulatory focus influences self-framing and risky decision making. Current Psychology. https://doi.org/:10.1007/s12144-019-00217-w
Salganik, M. J., \& Heckathorn, D. D. (2004). Sampling and estimation in hidden populations using respondent-driven sampling. In: R. M. Stolzenberg (Ed.), Sociological Methodology (pp. 193-239). Blackwell Publishing.

Strauss, K., Griffin, M. A., \& Parker, S. K. (2012). Future work selves: how salient hoped for identities motivate proactive career behaviors. Journal of Applied Psychology, 97(3), 580-598. https://doi.org/10.1037/a0026423

Tabachnick, B. G., \& Fidell, L. S. (2012). Using multivariate statistics (6 $6^{\mathrm{a}}$ ed.) Pearson Education, 2012.

Timmerman, M. E., \& Lorenzo-Seva, U. (2011). Dimensionality assessment of ordered polytomous items with parallel analysis. Psychological Methods, 16(2), 209-220. https:// doi.org/10.1037/a0023353

Wallace, J. C., Little, L. M., \& Shull, A. (2008). The moderating effects of task complexity on the relationship between regulatory foci and safety and production performance. Journal of Occupational Health Psychology, 13, 95-104. https://doi.org/10.1037/1076-8998.13.2.95

Van Beek, I., Toon W. T., Schaufeli, W.B, \& Brenninkmeijer, V. (2014). Heavy work investment: its motivational make-up and outcomes. Journal of Managerial Psychology, 29(1), 46-62. https://doi.org/10.1108/JMP-06-2013-0166

Van Vianen, A. E. M., Klehe, U. C., Koen, J., \& Dries, N. (2012). Career adaptabilities scale - Netherlands form: psychometric properties and relationships to abilities, personality, and regulatory focus. Journal of Vocational Behavior, 80, 716-724. https://doi.org/10.1016/j.jvb.2012.01.002

Vazquez, A. C. S., Magnan, E. dos S., Pacico, J. C., Hutz, C. S, \& Schaufeli, W. B. (2015). Adaptation and validation of the Brazilian version of the Utrecht Work Engagement Scale. Psico-USF, 20(2), 207-217. https://doi.org/10.1590/141382712015200202

Vohs, K. D., Baumeister, R. F., Schmeichel, B. J., Twenge, J. M., Nelson, N. M., Tice, D. M. (2014). Making choices impairs subsequent self-control: A limited-resource account of decision making, self-regulation, and active initiative. Motivation Science, 1(S), 19-42. https://doi.org/10.1037/ 2333-8113.1.S.19

Wallace, J. C., \& Chen, G. (2006). A multilevel integration of personality, climate, self-regulation, and performance. Personnel Psychology, 59, 529-557. https://doi.org/10.1111/ j.1744-6570.2006.00046.x

Zivnuska, S., Kacmar, M., \& Valle, M. (2017). The mechanisms of regulatory focus: Mindfulness, leader-member exchange, and motivational outcomes. Career Development International, 22(1), 37-49. https://doi.org/10.1108/CDI-07-2016-0120

Data de submissão: 02/10/2019 Primeira decisão editorial: 27/02/2020 Aceite em: 20/05/2020 